\title{
Effects of Distant Reiki On Pain, Anxiety and Fatigue in Oncology Patients in Turkey: A Pilot Study
}

\author{
Melike Demir ${ }^{1 *}$, Gulbeyaz Can², Ayhan Kelam³ $^{3}$, Aydın Aydıner ${ }^{3}$
}

\begin{abstract}
Background: Fatigue, stress and pain are common symptoms among cancer patients, affecting the quality of life. The purpose of the present study was to determine the effect of distant Reiki on pain, anxiety and fatigue in oncology patients. Materials and Methods: Participants in the control group received usual medical and nursing care during their stay. The intervention group received usual care plus five distant Reiki sessions, one each night for $30 \mathrm{~min}$. A face to face interview was performed and patient personal and illness related characteristics were evaluated using the Patient Characteristics form. Pain, stress and fatigue were evaluated according to a numeric rating scale. Results: The experimental group was predominantly composed of women $(\mathbf{7 1 . 4 \%})$, married individuals $(40 \%)$, and primary school graduates $(\mathbf{4 0 \%})$. The control group was predominantly male $(\mathbf{7 2 . 7 \%})$, married $(60 \%)$, and primary school graduates $(60 \%)$. The control group demonstrated greater levels of pain $(p=0.002)$, stress $(p=0.001)$ and fatigue $(p=0.001)$. The Reiki group pain score $(p<0.0001)$, stress score $(p<0.001)$ and fatigue score were also significantly lower. Conclusions: The results of this study indicate that Reiki may decreasepain, anxiety and fatigue in oncology patients
\end{abstract}

Keywords: Reiki - oncology - pain - anxiety - fatigue

Asian Pac J Cancer Prev, 16 (12), 4859-4862

\section{Introduction}

Complementary/alternative therapies described to five subcategory by the National Center for Complementary and Alternative Medicine (NCCAM) and Energy therapies are considered one of them (Coakley and Barron, 2012). The word Reiki is means life force energy and energy including the human body. Reiki restored and balanced to energy of body (Moore, 2005; Coakley and Barron, 2012;).

More than 1.2 million adult population reported that Reiki was a energy healing modalities in U.S. (Barnes et al., 2008). Reiki is a non invasive, inexpensive modality and it is offered to patients with cancer in hospitals and hospices throughout the country (Williams et al., 2009; Bossi et al., 2008, Burden et al., 2005).

Cancer has increasingly been recognized as a chronic illness. Cancer affects patients on physiological, social, and psychological levels (Tsang et al., 2007). Cancer treatment persist for long periods after diagnosis and treatment (Sikorskii et al., 2011).Cancer treatment cause lost of side effects. The pain, stress and fatigue is often seen side effects.

Fatigue, stress and pain is a common symptom among cancer patients, affecting the life style of the patient. In a study reported that fatigue was the most common problem $(48.5 \%)$ and the other problems are pain $(26.4 \%)$, stress $(24.8 \%)$, depression $(24.0 \%)$, or anxiety $(24.0 \%)$ (Carlson et al., 2004). Fatigue is affecting $50 \%$ to $70 \%$ of the population (Curt, 2000; Davidson et al., 2002; Carlson et al., 2004).

And then overall rates of stress were $35.1 \%$ in a large database of patients with cancer $(\mathrm{N}=9000)$ (Zabora et al., 2001 ) and $69 \%$ of cancer patients have reported that pain restricts their daily life activities (Breivik et al., 2009; Lee et al., 2014). In another study reported that pain occurs in $50 \%$ of patients with cancer undergoing treatment and more than $70 \%$ of patients experience pain towards the end of life (Davis et al., 2004). So that, the purpose of the study was to determine to effect of distant Reiki on pain, anxiety and fatigue in oncology patients.

\section{Materials and Methods}

\section{Setting and participants}

This randomized controlled trial was conducted in the Istanbul University Institute of Oncology in Turkey, from September 2013 to February 2014. The research group patients over the age of 18 , who were willing to be part of the study. All of them knew Turkish well, and had at least a primary school education.

The study population was composed of patients with cancer at any stage and receiving any kind of chemotherapy (CT). All participants were blinded as to whether or not they were in a Reiki group and were told prior to the start of the intervention that they may or may not receive noncontact Reiki. There were no statistical 
differences between the Reiki $(n=8)$ and No Reiki groups $(n=10)$.

\section{Intervention}

In distant Reiki, Reiki practitioner followed the traditional Usui Reiki protocol for distant healing. Reiki practitioner first undertake a name of patient and then send the healing energy to the patient. Participants in the control group received usual medical care during their stay. The Reiki group received usual care and five distant Reiki sessions, one each night in a day for $30 \mathrm{~min}$. A single Reiki practitioner located over $8 \mathrm{~km}$ away, who was trained in the Usui line of Reiki (Degre 2) and has been practising Reiki for over 4 years.

\section{Measures and instruments}

A face to face interview was performed. Depending on the inclusion criteria of the study patients were invited and informed consent was obtained. Patients' personal and illness related characteristics were evaluated using the Patient Characteristics form. Pain, stress and fatigue were evaluated according to a numeric rating scale.

The Patient Information Form was developed by the researchers contained 14 items that addressed both personal (e.g. age, income level, employment status,) and illness related characteristics (e.g. diagnosis, surgical therapy, radiation therapy).

Pain, stress, fatigue were evaluated according to a numeric rating scale. The VAS is a $10 \mathrm{~cm}$ line with an anchor at each end.

\section{Ethical considerations}

Research Committee of the Istanbul University Institute of Oncology approved this study. The patients were informed, and verbal consent was obtained.

\section{Statistical analysis}

The SPSS version 16 program was used for data analysis. Descriptive statistics, means, median, frequencies, and percentage were used to show the socio demographic and the illness or treatment related characteristics of the patients. Comparisons were made using t test for all statistical analyses, a 2 sided $\mathrm{p}$ value of less than 0.05 was considered statistically significant.

\section{Results}

Socio demographic characteristics of patients

The experimental group was predominantly women $(71.4 \%)$, married individuals $(40 \%)$, and primary school graduates (40\%). The patients' mean age was $38.62 \pm 19.50$ and most of the patients were Non Worker $(62.5 \%)$. The characteristics of the sample are shown in Table 1.

The control group was predominantly men (72.7\%), married individuals $(60 \%)$, and primary school graduates $(60 \%)$. The patients' mean age was $28.70 \pm 8.88$ and most of the patients were Worker $(62.5 \%)$. The characteristics of the sample are shown in Table 1. The two groups were similar with respect to all variables $(\mathrm{p}>0.05)$.

\section{Disease and treatment related characteristics}

The experimental group's median length of time since being given the diagnosis of cancer was 19.5 months ( $\bar{x}=19.5 \pm 24.56$, range $12-48$ months) and the median treatment cycle was $3.85(\bar{x}=3.85 \pm 2.79$, Range 2-10). The characteristics of the sample are shown in Table 1.

The control group's median length of time since being given the diagnosis of cancer was 21.6 months ( $\bar{x}=21.60 \pm 14.75$, range $12-60$ months) and the median treatment cycle was $3.60(\bar{x}=3.60 \pm 3.09$, Range 1-12). The

Table 1. Personal Characteristics and Disease Characteristics

\begin{tabular}{|c|c|c|c|c|}
\hline & \multicolumn{2}{|c|}{$\begin{array}{l}\text { Experimental } \\
\text { group }\end{array}$} & \multicolumn{2}{|c|}{$\begin{array}{l}\text { Control } \\
\text { group }\end{array}$} \\
\hline & $\mathrm{n}$ & $\%$ & $\mathrm{n}$ & $\%$ \\
\hline \multicolumn{5}{|l|}{ Gender } \\
\hline Females & 5 & $62.5^{*}$ & 2 & $20.0^{*}$ \\
\hline Males & 3 & $37.5^{*}$ & 8 & $80.0^{*}$ \\
\hline \multicolumn{5}{|l|}{ Marital status } \\
\hline Married & 4 & $50.0 *$ & 6 & $60.0^{*}$ \\
\hline Single & 4 & $50.0 *$ & 4 & $40.0^{*}$ \\
\hline \multicolumn{5}{|l|}{ Education level } \\
\hline Primary school & 2 & $25.0 *$ & 3 & $30.0^{*}$ \\
\hline High school & 3 & $37.5^{*}$ & 4 & $40.0^{*}$ \\
\hline University & 3 & $37.5^{*}$ & 3 & $30.0^{*}$ \\
\hline \multicolumn{5}{|l|}{ Occupation } \\
\hline Worker & 3 & $37.5 *$ & 6 & $60 *$ \\
\hline Non- Worker & 5 & $62.5^{*}$ & 4 & $40^{*}$ \\
\hline \multicolumn{5}{|l|}{ Diagnosis } \\
\hline Sarcoma & 3 & $37.5 *$ & 6 & $60 *$ \\
\hline Genito-urinary cancer & 3 & $37.5^{*}$ & 3 & $30 *$ \\
\hline Other & 2 & $25.0^{*}$ & 1 & $10 *$ \\
\hline \multicolumn{5}{|l|}{ Radiotherapy } \\
\hline No & 4 & $33.3 *$ & 8 & $66.7^{*}$ \\
\hline Yes & 4 & $66.7 *$ & 2 & $33.3^{*}$ \\
\hline \multicolumn{5}{|l|}{ Chemotherapy protocol } \\
\hline Paclitaxel+Cisplatin & 5 & $62.5 *$ & 3 & $30 *$ \\
\hline ifosfamide & 3 & $37.5 *$ & 7 & $70 *$ \\
\hline
\end{tabular}

* Percent of intra-group

Table 2. The Effect of Distant Reiki on Pain, Anxiety and Fatigue

\begin{tabular}{|c|c|c|c|c|c|c|c|c|c|c|c|c|}
\hline & \multicolumn{4}{|c|}{ Experimental } & \multirow[t]{3}{*}{$\mathrm{t}$} & \multirow[t]{3}{*}{$\mathrm{p}$} & \multicolumn{4}{|c|}{ Control } & \multirow[t]{3}{*}{$\mathrm{t}$} & \multirow[t]{3}{*}{$\mathrm{p}$} \\
\hline & \multicolumn{2}{|c|}{ Pre-test } & \multicolumn{2}{|c|}{ Last Test } & & & \multicolumn{2}{|c|}{ Pre-test } & \multicolumn{2}{|c|}{ Last Test } & & \\
\hline & $\overline{\mathrm{x}}$ & $\pm \mathrm{sd}$ & $\overline{\bar{x}}$ & $\pm \mathrm{sd}$ & & & $\overline{\mathrm{X}}$ & $\pm \mathrm{sd}$ & $\overline{\bar{X}}$ & $\pm \mathrm{sd}$ & & \\
\hline Pain & 3.62 & 1.06 & 2.00 & 0.75 & 9.66 & $<0.0001$ & 3.70 & 2.75 & 4.20 & 2.69 & 4.25 & 0.002 \\
\hline Stress & 4.25 & 1.98 & 1.37 & 0.74 & 6.06 & 0.001 & 4.30 & 2.89 & 4.90 & 2.42 & 4.73 & 0.001 \\
\hline Fatigue & 2.87 & 0.83 & 1.75 & 0.70 & 9.74 & 0.001 & 2.70 & 1.76 & 3.80 & 2.25 & 4.83 & 0.001 \\
\hline
\end{tabular}


characteristics of the sample are shown in Table 1. The two groups were similar with respect to all variables $(p>0.05)$.

\section{The degree of pain}

In the first assessment experimental group's mean pain score were $3.62 \pm 1.06$ and control mean pain score were 3.70 \pm 2.75 . After Reiki practice experimental group's mean pain score $2.00 \pm 0.75$. We make no attempt to control group. We assessment control group's mean pain score and experimental group's pain score at the same time and then we have seen that control group's mean pain score increased (4.20 \pm 2.69$)$. The control group was observed that increased levels of pain $(\mathrm{p}=0.002)$, it was observed that the experimental group decreased pain intensity ( $\mathrm{p}$ $<0.0001$ ) (Table 2).

\section{The degree of stress}

In the first assessment experimental group's mean stress score were $4.25 \pm 1.98$ and control mean stress score were $4.30 \pm 2.89$. After Reiki practice experimental group's mean stress score decreased $(1.37 \pm 0.74)$ and control group's mean stress score increased (4.90 \pm 2.42$)$. The control group was observed that increased levels of stress $(\mathrm{p}=0.001)$, but the experimental group's stress score $(\mathrm{p}<0.001)$ (Table 2).

\section{The degree of fatigue}

In the first assessment experimental group's mean fatigue score were $2.87 \pm 0.83$ and control mean fatigue score were $2.70 \pm 1.76$. After Reiki practice experimental group's mean fatigue score decreased $(1.75 \pm 0.70)$ and control group's mean fatigue score increased (3.80 \pm 2.25$)$. The control group was observed that increased levels of fatigue ( $\mathrm{p}=0.001)$, But Reiki group's fatigue levels decreased $(\mathrm{p}<0.001)$ (Table 2).

\section{Discussion}

Cancer has increasingly been recognized as a chronic illness involving a series of changing threats and difficulties (Tsang et al., 2007) so that fatigue, stress and pain is a common symptom among cancer patients. Recent systematic review reported that Reiki can be effective treatment (Lee et al., 2008). Because of this study's aim was to determine to effect of distant Reiki on pain, anxiety and fatigue in oncology patients.

In our study, Reiki groups' pain score decreased and non Reiki groups pain score increased. So the study showed that Reiki decreased the pain level. A study with cancer patients showed that Reiki is not effective on pain (Olson et al., 2003). A study showed that to Reiki reduction of $50 \%$ in mean scores of pain after each chemotherapy treatment (Birocco et al., 2012). Another study reported that Reiki decreased pain, depression, and anxiety in chronically ill people (Dressin and Singg, 1998). These results suggest that Reiki treatments are moderately effective at reducing pain levels.

We found that Reiki decreased the stress score in oncology patients. A study demonstrated that physical stress decreased after 20 minutes of Reiki; while mental stress was not reduced by other therapies (Witte and
Dundes, 2001). Another study reported that distance Reiki reduction depression and stress (Shore, 2004). As same as another study reported that Reiki reduced physiological effects of stress (Baldwin et al., 2008). In another study reported that Reiki there were persuasive reductions in the total Stress Scale score which were greater than in the Non Reiki group (Bowden et al., 2010). This preliminary study and our study supports Reiki may be useful in reducing the anxiety, stress response and promoting relaxation (Vitale, 2007; Miles, 2003; Bullock, 1997; Barnett and Chambers, 1996).

Our data showed that Reiki decreased the fatigue level in oncology patients who are lying in the hospital for treatment. A study reported that fatigue decreased within the Reiki session over seven treatments compared with the rest session, but Reiki wasn't significantly decreased fatigue level (Tsang et al., 2007). There are a little study so there is a need for future studies.

This study is limited by small sample sizes, lack of comparison groups. Although patients are admitted to Istanbul University Institute of Oncology from all regions of Turkey, the study results may not be generalizable to all Turkish cancer patients.

In our study showed that Reiki is a effective management on pain, stress and fatigue. There are only a few studies with Reiki and needs to be done a lot of study on this subject in the future. There is limited evidence as to their efficacy for symptom management, including reducing pain and fatigue but Deng et al. recommendation Reiki for reducing anxiety, pain and fatigue (Deng et al., 2009).

\section{References}

Baldwin AL, Wagers C, Schwartz GE (2008). Reiki improves heart rate homeostasis in laboratory rats.J Altern Complement Med, 14, 417-22.

Barnes PM, Bloom B, Nahin RL (2008). Complementary and alternative medicine use among adults and children: United States, 2007. Natl Health Stat Rep, 12, 1-24.

Barnett L, Chambers M (1996). Reiki energy medicine: bringing healing touch into home, hospital and hospice. Rochester, VT: Healing Arts Press.

Birocco N, Guillame C, Storto S, et al (2012). The Effects of Reiki Therapy on Pain and Anxiety in Patients Attending a Day Oncology and Infusion Services Unit. Am J Hospice Palliat Med, 29, 290-4.

Bossi LM, Ott MJ, DeCristofaro S (2008). Reiki as a clinical intervention in oncology nursing practice. Clin J Oncol Nurs, 12, 489-94.

Bowden D, Goddard L, Gruzelier J (2010). A randomised controlled single-blind trial of the effects of Reiki and positive imagery on well-being and salivary cortisol. Brain Research Bulletin, 81, 66-72.

Breivik H, Cherny N, Collett F, et al (2009). Cancer-related pain: a pan European survey of prevalence, treatment, and patient attitudes. Ann Oncol, 20, 1420-1433.

Bullock M (1997). Reiki: a complementary therapy for life. Am $J$ Hosp Palliat Care, 14, 31-33.

Burden B, Herron-Marx S, Clifford C (2005). The increasing use of Reiki as a complementary therapy in specialist palliative care. Int J Palliat Nurs, 11, 248-53.

Carlson LE, Angen M, Cullum J, et al (2004). High levels of untreated distress and fatigue in cancer patients. Br J Cancer, 
90, 2297-304.

Coakley A and Barron A (2012). Energy Therapies in Oncology Nursing. Seminars Oncol Nur, 28, 55-63.

Curt GA (2000). Impact of fatigue on quality of life in oncology patients. Semin Hematol, 37, 14-17.

Davidson JR, MacLean AW, Brundage MD, et al (2002). Sleep disturbance in cancer patients. Soc Sci Med, 54, 1309-21.

Davis MP, and Walsh D (2004). Epidemiology of cancer pain and factors influencing poor pain control. Am J Hosp Palliat Care, 21, 137-142.

Deng G, Frenkel M, Cohen L, et al (2009). Evidence-based clinical practice guidelines for integrative oncology: complementary therapies and botanicals. J Soc Integrative Oncol, 7, 85-120.

Dressin LJ, and Singg S (1998). Effects of Reiki on pain and selected affective and personality variables of chronically ill patients. Subtle Energies Energy Med, 9, 51-82.

Lee MS, Pittler MH, Ernst E (2008). Effects of Reiki in clinical practice: A systematic review of randomized clinical trials. International J Clin Pract, 62, 947-954.

Miles P (2003). Preliminary report on the use of Reiki HIVrelated pain and anxiety. Altern Ther Health Med, 9, 36.

Moore A (2005). Reiki energy medicine: enhancing the healing process. Integrative Medicine Quarterly News, 2, 1-5.

Olson K, Hanson J, Michaud M (2003). Other complementary therapies, Reiki effects on pain and quality of life in advanced cancer patients. J Pain Symptom Manage, 26, 990-997.

Shore A (2004). Long-term effects of energetic healing on symptoms of psychological depression and self-perceived stress. Alt Ther, 10, 42-48.

Sikorskii A, Wyatt G, Siddiqi A, Tamkus D (2011). Recruitment and early retention of women with advanced breast cancer in a complementary and alternative medicine trial. Evid Based Complement Alternat Med Article, 734517, 7.

Tsang KL, Carlson LE, Olson K (2007). Pilot crossover trial of Reiki versus rest for treating cancer-related fatigue. Integr Cancer Ther, 6, 25-35.

Vitale AT (2007). An integrative review of Reiki touch therapy research. Holist Nurs Pract, 21, 167-79.

Williams AM, Davies A, Griffiths G (2009). Facilitating comfort for hospitalized patients using non-pharmacological measures: preliminary development of clinical practice guidelines. Int J Nurs Pract, 15, 145-55.

Witte D, Dundes L (2001). Harnessing life energy or wishful thinking? Reiki, placebo Reiki, meditation, and music. Altern Complement Ther, 7, 304-309.

Zabora J, Brintzenhofeszoc JK, Curbow B, et al (2001). The prevalence of psychological distress by cancer site. Psychooncol, 10, 19-28. 